\title{
TWO CHARACTERIZATIONS OF LINEAR BAIRE SPACES
}

\author{
STEPHEN A. SAXON ${ }^{1}$
}

\begin{abstract}
The Wilansky-Klee conjecture is equivalent to the (unproved) conjecture that every dense, 1-codimensional subspace of an arbitrary Banach space is a Baire space (second category in itself). The following two characterizations may be useful in dealing with this conjecture: (i) A topological vector space is a Baire space if and only if every absorbing, balanced, closed set is a neighborhood of some point. (ii) A topological vector space is a Baire space if and only if it cannot be covered by countably many nowhere dense sets, each of which is a union of lines (1-dimensional subspaces). Characterization (i) has a more succinct form, using the definition of Wilansky's text [8, p. 224]: a topological vector space is a Baire space if and only if it has the $t$ property.
\end{abstract}

Introduction. The Wilansky-Klee conjecture (see [3], [7]) is equivalent to the conjecture that every dense, 1-codimensional subspace of a Banach space is a Baire space. In [4], [5], [6], [7] it is shown that every countable-codimensional subspace of a locally convex space which is "nearly" a Baire space is, itself, "nearly" a Baire space. (Theorem 1 of this paper indicates how "nearly Baire" a barrelled space is: Wilansky's class of W-barrelled spaces [9,p. 44] is precisely the class of linear Baire spaces.) The theorem [7] that every countable-codimensional subspace of an unordered Baire-like space is unordered Baire-like is the closest to an affirmation of the conjecture. (A locally convex space is unordered Baire-like if it cannot be covered by countably many rare [nowhere dense], balanced, convex sets.) The two characterizations in this note, of some independent interest, seem also likely aids for tackling the Wilansky-Klee conjecture. (E.g., see Remarks (a).)

Presented to the Society, January 15, 1974; received by the editors January 29, 1973.

AMS (MOS) subject classifications (1970). Primary 46A15; Secondary 46A07.

Key words and phrases. Wilansky-Klee conjecture, topological vector spaces, Baire spaces, absorbing, balanced, closed set, unordered Baire-like spaces.

1 The author wishes to thank Professors V. Klee, A. Todd and A. Wilansky for their recent helpful personal communications. 
Definitions. The definitions of Horváth's text [2] are generally adopted here. In particular, if $A, B$ are subsets of a vector space $E$, we say $B$ is balanced if $\lambda B \subset B$ whenever $|\lambda| \leq 1$, and $A$ is absorbing if, given $y$ in $E$, there corresponds a $\delta>0$ such that $\lambda y \in A$ whenever $|\lambda| \leq \delta$.

Theorem 1 (cf. 2.1 Theorem of [7]). A topological vector space $E$ is a Baire space if and only if every absorbing, balanced, closed set $B$ is a neighborhood of some point.

Proof. If $B$ is rare and absorbing, then $E=\bigcup_{n=1}^{\infty}(n B)$ and each $n B$ is rare, implying $E$ is not Baire.

Conversely, if $E$ is not Baire, then there exist $x$ in $E$ and a balanced, closed neighborhood $U$ of zero such that $x$ is not in $U$. Let $V$ be a balanced, closed neighborhood of zero such that $V+V \subset U$ and let $\left\{A_{n}\right\}$ be a sequence of rare, closed sets whose union is $V$. We assume $E$ is a complex vector space. (The real case is similar but easier.) Define

$$
B_{n}=\bigcup_{k=0}^{n-1} e^{2 k \pi i / n}\left(A_{1} \cup \cdots \cup A_{n}\right) \quad \text { for } n=1,2, \cdots \text {. }
$$

[If $E$ were real, we would let $B_{n}=\left(A_{1} \cup \cdots \cup A_{n}\right) \cup(-1)\left(A_{1} \cup \cdots \cup A_{n}\right)_{\cdot}$ ] We show that $H=\bigcup_{n=1}^{\infty}\left((1 / n) B_{n}\right)$ is absorbing and rare. Let $y$ be in $E$, and let $\mathrm{sp}(\{y\})$ denote the span of $\{y\}$. Since $V$ is absorbing, balanced and closed, $\operatorname{sp}(\{y\}) \cap V$ is a Baire space and there exist $p$ a positive integer, $\delta>0$, and $z$ a complex number such that

$$
\alpha y \in A_{p} \quad \text { for }|\alpha-z| \leq \delta .
$$

Since $e^{i \theta}$ is uniformly continuous on $0 \leq \theta \leq 2 \pi$, there is an integer $q \geq p$ such that

$$
\left|e^{i \theta_{1}}-e^{i \theta_{2}}\right| \cdot|z| \leq \delta / 2 \text { for }\left|\theta_{1}-\theta_{2}\right| \leq 2 \pi / q .
$$

Suppose $n \geq q$. Then given $\theta, 0 \leq \theta \leq 2 \pi$, there exists an integer $k, 0 \leq k$ $\leq n-1$, such that $|\theta-2 k \pi / n| \leq 2 \pi / n \leq 2 \pi / q$, so that $(* *)$ implies

$$
\left|e^{i \theta}-e^{2 k \pi i / n}\right| \cdot|z| \leq \delta / 2 .
$$

Since $B_{n} \supset \bigcup_{k=0}^{n-1} e^{2 k \pi i / n} A_{p}$, (*) and (***) imply that $(* * * *) \quad \lambda y \in B_{n} \quad$ whenever $|z|-\delta / 2 \leq|\lambda| \leq|z|+\delta / 2$.

Fix $N \geq q$ such that $|z| /(N+1) \leq \delta / 2$. Suppose, for some $n \geq N$, $\gamma$ satisfies $|z| / n \geq|\gamma| \geq|z| /(n+1)$. Then 


$$
\begin{aligned}
\frac{1}{n}\left(|z|-\frac{\delta}{2}\right) & =\frac{|z|}{n}-\left(\frac{\delta}{2}\right)\left(\frac{1}{n}\right) \leq \frac{|z|}{n}-\frac{|z|}{n+1} \cdot \frac{1}{n} \\
& =\frac{|z|}{n+1} \leq|\gamma| \leq \frac{|z|}{n} \leq \frac{1}{n}\left(|z|+\frac{\delta}{2}\right)
\end{aligned}
$$

so that $\gamma y \in\left((1 / n) B_{n}\right)$ by $(* * * *)$. Hence $\gamma y \in H$ for $0 \leq|\gamma| \leq|z| / N$ (zero is obviously in $H$ ) and $H$ absorbs $y$ if $z \neq 0$. If $z=0,(* * * *)$ implies $H$ absorbs $y$. Since $y$ was chosen arbitrarily, $H$ is absorbing.

We see that $H$ is rare, exactly as in [7]: if $H^{-}$contains some open neighborhood $W$ of a point $y$, then, since $\bigcup_{n<k}\left((1 / n) B_{n}\right)$ is a finite union of rare sets and hence rare, we have

$$
W \subset\left[\bigcup_{n \geq k}\left(\frac{1}{n} B_{n}\right)\right]^{-} \subset \frac{1}{k} V
$$

for $k=1,2, \cdots$. Also, there is some $\delta>0$ such that $y \pm \delta x \in W$. But this would imply that

$$
2 \delta x=(y+\delta x)-(y-\delta x) \in V / k+V / k \subset U / k
$$

for $k=1,2, \cdots$, a contradiction since $2 \delta x \notin(1 / k) U$ whenever $1 / k \leq 2 \delta$. ( $U$ is balanced.) Thus $H$ is rare.

Finally, we let $B$ be the closure of the balanced core of $H[2, \mathrm{p} .80]$ to obtain an absorbing, balanced, closed, rare set, which completes the proof.

Remarks. (a) Application of Theorem 1 facilitates the proof of Theorem 5.1 in [7]: if $B$ is an absorbing, balanced, closed set in a closed, 1-codimensional subspace $M$ of a topological vector space $E$ and $x \in E \sim M$, then $B^{\prime}=B+\{\lambda x:|\lambda| \leq 1\}$ is clearly absorbing, balanced and closed in $E$, and if $B$ is rare in $M$, so is $B^{\prime}$ in $E$.

(b) A balanced, convex set has an interior point if and only if it is a neighborhood of zero. Thus Theorem 1 invites comparison of linear Baire spaces with the several important classes of locally convex spaces defined by prescribing what sorts of absorbing, balanced, convex sets should be neighborhoods of zero. (See [2, Chapter 3].) For example, a locally convex space is (Baire) [barrelled] if and only if every (absorbing, balanced, closed) [and convex] set has an interior point. In the same vein, a locally convex space is Baire if and only if

$(\dagger)$ it cannot be covered by countably many rare, balanced sets.

Here $(\dagger)$ differs from the definition of "unordered Baire-like" only in the omission of the word "convex".

(c) By Theorem 1, if a space is not Baire, it can be covered by an in- 
creasing sequence of rare, balanced sets. (Cf. definition of "Baire-like" in [6].) In [1, p. 145, Exercise 8], Grothendieck showed a certain normed, barrelled space is not Baire by covering it with an increasing sequence of rare sets, each a union of 1-dimensional subspaces; i.e., each closed under scalar multiplication. The import of Theorem 2, below, is that every nonBaire space can be so covered. However, we cannot, in general, additionally require the sets to be closed under vector addition, since there are quasiBaire spaces [5] which are not Baire (nor even Baire-like).

Theorem 2. A topological vector space $E$ is a Baire space if and only if it cannot be covered by an increasing sequence of rare sets, each of which is closed under scalar multiplication.

Proof. Let $x, U, V, B$ be as in the proof of Theorem 1. Let $K_{n}=$ $\{y \in B: n y \notin V\}$, and let $L_{n}$ be all scalar multiples of elements of $K_{n}(n=$ $1,2, \cdots)$. Now $w \in L_{n}$ implies $w=a y$, where $y \in B$ and $y \notin(1 / n) V$. If also $w \in(1 / n) V$, then $|\alpha|<1$, since $(1 / n) V$ is balanced, and therefore $w \in B$, since $B$ is balanced. That is, $L_{n} \cap((1 / n) V)$ is contained in the rare set $B$, and hence is rare. Indeed, $L_{n}$ is rare, for if $L_{n}$ were dense in a nonempty open set then $L_{n} \cap((1 / n) V)$ would be also; scalar multiplication is continuous, $L_{n}$ is closed under scalar multiplication, and $(1 / n) V$ is a neighborhood of zero $(n=1,2, \cdots)$. Define $L_{0}=\{y \in V: n y \in V \quad$ for $n=1,2, \cdots\}$. $L_{0}$, which may or may not be empty, is closed under scalar multiplication. Now $L_{0} \cup L_{1} \cup L_{2} \cup \cdots$ is closed under scalar multiplication and contains the absorbing set $B \subset V$, and hence is all of $E$. To see that the sequence $\left\{S_{n}\right\}$ fulfills the requirements of the theorem, where $S_{n}=L_{0} \cup L_{1} \cup \cdots \cup L_{n}$ $(n=1,2, \cdots)$, one needs yet to observe that $L_{0}$ is rare. (Finite unions of rare sets are rare.) But $F=L_{0}+L_{0}$ is a linear subspace contained in $V+$ $V \subset U$. Hence $F^{-} \subset U$ is a closed, proper subspace $(x \notin U)$, which means that $F^{-}$is rare; thus $L_{0} \subset F^{-}$is also rare.

\section{REFERENCES}

1. A. Grothendieck, Espaces vectoriels topologiques, 3rd. ed, São Paulo, Sociedade de Mathemática de São Paulo, 1964.

2. J. Horváth, Topological vector spaces and distributions. Vol. I, AddisonWesley, Reading, Mass., 1966. MR 34 \#4863.

3. V. Klee and A. Wilansky, Research problems, \#13, Bull. Amer. Math. Soc. 28 (1966), p. 151.

4. M. Levin and S. Saxon, Every countable-codimensional subspace of a barrelled space is barrelled, Proc. Amer. Math. Soc. 29 (1971), 91-96. MR 43 \#6691. 
5. S. Saxon, $(L F)$-spaces, quasi-Baire spaces, and the strongest locally convex topology (to appear).

6. - Nuclear and product spaces, Baire-like spaces, and the strongest locally convex topology, Math. Ann. 197 (1972), 87-106.

7. A. Todd and S. Saxon, A property of locally convex Baire spaces, Math. Ann. 206 (1973), 23-34.

8. A. Wilansky, Functional analysis, Blaisdell, New York, 1964. MR 30 \#425.

9. - Topics in functional analysis, Lecture Notes in Math., no. 45, Springer-Verlag, Berlin and New York, 1967. MR 36 \#6901.

DEPARTMENT OF MATHEMATICS, UNIVERSITY OF FLORIDA, GAINESVILLE, FLORIDA 32611 